\title{
Generation and Detection of Watermarks Derived from Chaotic Functions
}

\author{
Aidan Mooney and John G. Keating \\ Department of Computer Science, N.U.I. Maynooth, Maynooth, \\ Co. Kildare, Ireland.
}

\begin{abstract}
A digital watermark is a visible, or preferably invisible, identification code that is permanently embedded in digital media, to prove owner authentication thereby providing a level of document protection. In this paper, we review several approaches for the generation of watermarks using chaotic functions, and in particular, the logistic chaotic function. Using this function, in conjunction with seed management, it is possible to generate chaotic sequences that may be used to create highpass or lowpass digital watermarks. A slight change in the initial conditions will quickly lead to a significant change in the subsequent states of the system, and thus will generate substantially different watermarks. This technique has been shown to offer an added security advantage over the more traditionally generated watermarks created from pseudorandom sequences, in that only the function seed needs to be stored. It also has the advantage that, through examination of the theoretical properties of the function, it is possible to choose seeds that lead to robust, lowpass watermarks. We review various detection techniques including correlation and statistical methods, and present an analysis of the impact of noise present in a model optical detector. The logistic function presented in this paper is ill defined for certain seed values and has not been fully investigated for the purpose of watermark generation. We consider the impact of the theoretical properties of the logistic function for several chaos-based watermark generation techniques, in particular, their highpass and lowpass properties, which when embedded in digital media, are suitable for correlation and statistical based detection methods.
\end{abstract}

\section{INTRODUCTION}

Digital Watermarking provides a means of inserting additional information into digital documents (cover documents). This extra information may be used to provide a certain degree of protection of the document from malicious attack. This information is usually a piece of identifying information which may be used at a later date to prove ownership. The original document owner can prove ownership of attacker copies by proving that the copy contains their original watermark. The cover image used in this paper is the widely used "Lena" image of size $256 \times 256$, shown in Fig. 1(a).

Numerous watermark generation techniques have been proposed to date, ranging from the use of personal logos to the use of pseudorandom sequences of numbers ${ }^{1}$. The use of chaotic functions for the generation of watermarks has also been proposed by Pitas et al. ${ }^{2-4}$. The functions used included ones which produced Markov Maps and Bernoulli Maps. This type of watermark generation schemes require two values, the initial value and the function seed, in order to recreate the same watermark at a later stage. An advantage of these watermarks is their robustness to lowpass attacks. Typically these watermarks are distributed over the full cover image using an additive or multiplicative technique.

Further author information:

E-mail: amooney@cs.may.ie, jkeating@cs.may.ie 



Figure 1. (a) Cover Image - Lena (b) Bifurcation Diagram for the Logistic Map.

\section{WATERMARK GENERATION TECHNIQUES}

In this paper, we examine watermarks that are generated using chaotic functions. A chaotic function is a function which is sensitive to initial conditions, is unpredictable, indecomposable and yet contains regularity ${ }^{5}$. The motivation for using a chaotic function to generate a watermark lies in the fact that a single variable, $a$, seeding the chaotic function, will always result in the same output (mapping) when certain constraints or initial conditions are placed on the mapping. Such watermarks have robustness properties that may be analysed analytically. A chaotic map is derived from a chaotic sequence fully described by the map $\left\{y_{j}: y_{j}=f\left(y_{j-1}, a\right)\right\}$ and an initial condition $y_{0}$.

The use of chaotic watermarks has been shown to offer an added security advantage over the more traditionally generated watermarks created from pseudorandom sequences, in that only the function seed needs to be stored. It also has the advantage that, through examination of the theoretical properties of the function, it is possible to choose seeds that lead to robust, lowpass watermarks.

\subsection{Logistic Equation}

The function used in this paper for the generation of sequences which are transformed into watermarks is the logistic difference equation:

$$
y_{n+1}=a y_{n}\left(1-y_{n}\right)
$$

where $a$ is the function 'seed' and $y_{n}$ is the current value of the mapping in time ${ }^{6}$.

The value $y_{n+1}$ is dependant on its current density $y_{n}$. For low values of $a, y_{n}$ eventually converges to a single number as $n$ goes to infinity. When $a$ equals $3.0, y_{n}$ no longer converges - it oscillates between two values. This characteristic change in behaviour is called a bifurcation, as shown in Fig. 1(b). Increasing the value of $a$ even further causes $y_{n}$ to oscillate between not two, but four values. As $a$ increases, $y_{n}$ goes through bifurcations of period $2^{n}$ and eventually becomes chaotic. These period-doubling bifurcations are called pitchfork bifurcations, due to their resemblance to a pitchfork. When the value of $a \approx 3.57, y_{n}$ neither converges or oscillates - its value becomes completely random. For values of $a$ larger than 3.57 , the behaviour is mainly chaotic ${ }^{6}$.

When the logistic equation is seeded with a value $3.57 \leq a \leq 4.0$ (the chaotic region), and the map iterated, chaotic behaviour is witnessed, in general, and it is this feature of the logistic equation that we utilise to generate watermarks. The trajectory's produced for maps of this equation will differ greatly even for small differences in the seed value for the equation, provided the seed is within the chaotic region. The $1 \mathrm{D}$ sequence generated by iterating this map is a sequence of real numbers between 0 and 1 and are converted to one of two values:

$$
y_{n}= \begin{cases}0 & y_{n} \leq 0.5 \\ 1 & y_{n}>0.5\end{cases}
$$


where 0.5 is a threshold and $y_{n}$ is the $n^{t h}$ value in the sequence generated by the equation.

This sequence needs to be converted into a $2 \mathrm{D}$ sequence which one may use as a watermark. In this paper, watermarks are generated which are the same size as the image which will contain the watermark. A scanning technique, known as Peano Scanning, is used to determine the order which the pixels of the sequence get embedded into the watermark being generated. Peano Scanning is preferable to the more conventional Raster Scanning technique in that its scanning is not predictable and can produce many variations of scanning within the same image. The only drawback with using Peano Scanning is that it will only operate on images whose height and width are multiples of 2 . The Peano scanning order always moves to a neighbouring pixel, but the pattern may appear in different orientations depending on the starting pixel with the scanning routine ${ }^{7,8}$.

\subsection{Skew Tent Map}

Skew Tent Maps have previously been used in the generation of watermarks ${ }^{9,10}$. The skew tent map can be expressed as:

$$
\tau(x)=\left\{\begin{array}{l}
\left(\frac{1}{\alpha}\right) x \quad, \quad[0,1] \rightarrow[0,1] \\
\tau(x)=\left(\frac{1}{\alpha-1}\right) x+\left(\frac{1}{1-\alpha}\right) \quad, \quad \alpha<x \leq 1
\end{array}, \alpha \in[0,1]\right.
$$

By varying the parameter $\alpha$, either highpass $(\alpha<0.5)$, or lowpass $(\alpha>0.5)$ sequences can be produced ${ }^{2}$. Fig. 2 shows the Skew Tent Map in the case where $\alpha=0.9$. Fig. 3 shows the watermarks produced when (a) $\alpha=0.1$ and (b) $\alpha=0.9$, and Peano Scanning used to determine the pixel location. For $\alpha=0.5, \tau(x)$ becomes the tent map.
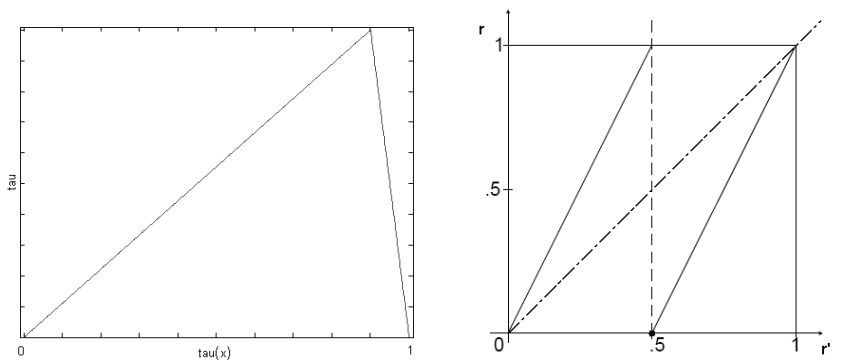

Figure 2. (a) Skew Tent Map when $\alpha=0.9$ (b) Bernoulli Shift Map.
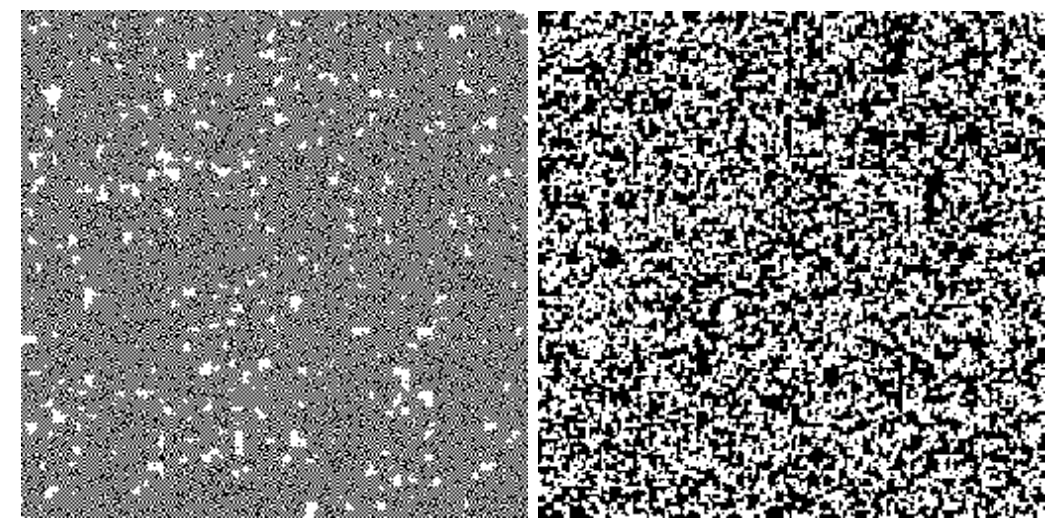

Figure 3. Watermark produced when (a) $a=0.1$ and (b) $a=0.9$ for the Skew Tent Map. 


\subsection{Bernoulli Shift Maps}

Bernoulli Shift Maps have previously been used in the generation of watermarks ${ }^{4,11}$. $n$-way Bernoulli shifts $B_{n}(p)$ are chaotic maps defined in the interval $[0,1]$ through the following expression:

$$
r^{\prime}=B_{n}(r)=n r(\bmod 1)
$$

When the value of $n=2$ the mapping is referred to as a Bernoulli Shift Map. Fig. 2(b) shows the Bernoulli Shift Map with the interval being the threshold value 0.5. The concept behind the Bernoulli Shift is from a more general map, known as a shift map defined by the equation:

$$
D(x)= \begin{cases}2 x & \text { if } \quad 0 \leq r<\frac{1}{2} \\ 2 x-1 & \text { if } \quad \frac{1}{2} \leq r<1\end{cases}
$$

As in the case of other chaotic maps, a chaotic watermark sequence is generated by the recursive application of the Bernoulli Shift Map. The output from a Bernoulli Shift Map is indistinguishable from noise even though the process is completely deterministic.

\section{PROPERTIES OF THE LOGISTIC FUNCTION}

Skew Tent Maps and Bernoulli Maps are well defined and have been studied in depth for the purpose of watermark generation. However no such analysis has been performed on the logistic map. In the following section we attempt to provide an overview of our work in this area.

\subsection{Periodic windows embedded in the chaotic regions of the logistic map}

There are certain regions of the logistic map where chaotic behaviour is not observed. It is known that no chaotic behaviour is observed for values of the seed $a<3.5699$. This point in the function is known as the accumulation point. Beyond this point the periodicity witnessed before it gives way to chaos. Within this chaotic region $(3.5699 \leq a \leq 4)$ there are periodic regions present. These occur at the bifurcation zones of the chaotic region of the map, for example, sizeable regions exist when $3.627 \leq a \leq 3.634$ (period-6), $3.739 \leq a \leq 3.744$ (period-5) and $3.829 \leq a \leq 3.856$ (period-3).

The most prominent of these regions is the period-3 window starting at $a=\sqrt{(8)}+1$. Once the period length 3 has been observed it is known that all possible periods occur ${ }^{12}$. In the period- 3 window the period doubling occurs again, leading to orbits of period length $6,12,24,48, \ldots$ and so on until another period-3 occurs and so forth. The bifurcation diagram in this period-3 window may be seen in Fig. 4(a) and in Fig. 4(b) where an enlarged version of the period-3 window is shown. Note, the similarity between this region and the map around the accumulation point.

If the watermark generation process is seeded with a value of $3.829 \leq a \leq 3.856$ periodic behaviour is witnessed. In this region the system takes on one of only three values. The sequences produced are periodic, and also predictable and therefore are not suitable for watermark generation. An example of one such watermark $(a=3.84)$ using Peano Scanning to determine the pixel locations is shown in Fig. 5(a), and one not using Peano Scanning is shown in Fig. 5(b). These figures illustrate that periodic predictable sequences are produced even in the so-called "chaotic region". It can be seen that there is a pattern developing within the watermark and this is due to the predictability of the map at this location. Similar results are found for each of the periodic regions of the map, indicated previously.

\subsection{Fixed Points}

There are other stable states within this chaotic region $(\approx 3.57 \leq a \leq 4)$ which are not suitable for use in watermark generation. These stable states occur when a trajectory arrives at a fixed point of the logistic map. A fixed point occurs whenever $y_{n+1}=y_{n}$. Solving this equation (and dropping the subscript) will give the values of these fixed points.

$$
\begin{aligned}
y[1-a(1-y)] & =y(1-a+a y) \\
& =a y\left[y-\left(1-a^{-1}\right)\right]=0
\end{aligned}
$$



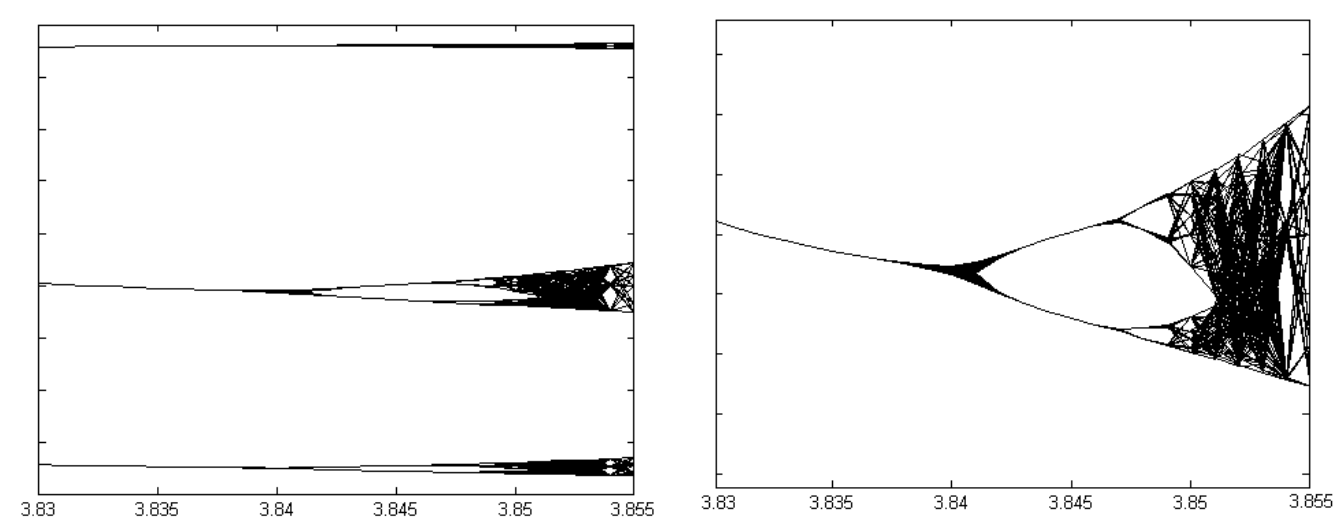

Figure 4. (a) Bifurcation Diagram for the period-3 window and (b) Enlarged version of the bifurcation Diagram for the Logistic Map in a period-3 window.


Figure 5. Watermark generated when (a) $a=3.84$ and incorporating Peano Scanning and (b) $a=3.84$ not incorporating Peano Scanning.

Therefore the fixed points are: ${ }^{13}$

$$
y_{0}=0 \quad \text { and } \quad y_{0}=1-\mathrm{a}^{-1}
$$

and for the case of $a=4$ the two fixed points which satisfy Eq. 7 are $y_{0}=0$ and $y_{0}=\frac{3}{4}$.

When deciding on a Function seed for the logistic equation the user should be fully aware of the chaotic region of the map produced. They should be aware that although the section of the map from $3.57 \leq a \leq 4$ is commonly referred to as the "chaotic region" there are areas of this region that should be avoided. These occur when windows of periodic activity occur such as windows of period-3, period-5 and period- 6 as discussed in Section 3.1.

Fixed states of a trajectory should also be avoided when selecting the function seed. If one of these fixed states is used to generate a watermark, there is no density variation (i.e. either a completely black or white watermark) which renders it unsuitable. There are two fixed points for each value of the function seed and they are known to occur when the initial value $y_{i}=0$ and $y_{i}=1-a^{-1}$. An example of a fixed state occurs when $a=4$ and with an initial value of $x_{0}=0$ or $x_{0}=\frac{3}{4}$.

\section{WATERMARK EMBEDDING}

Watermark Embedding is the process of inserting the generated watermark into some cover data, in this case an image. Watermarking may also be used for audio and video. The generated watermark is embedded in the 
spatial domain or in some transform domain (DCT, DFT, DWT) in one of two manners: additive technique or multiplicative technique. The additive technique is given by:

$$
m_{n}=j_{n}+\gamma y_{n}
$$

and the multiplicative technique is given by:

$$
m_{n}=j_{n}+\gamma j_{n} y_{n}
$$

where $m_{n}$ is the $n^{t h}$ pixel of the watermarked image $M, j_{n}$ is the $n^{t h}$ pixel of the cover image $J, y_{n}$ is the $n^{t h}$ pixel of the watermark $Y$ and $\gamma$ is known as the embedding factor and controls the watermark strength. The multiplicative watermark embedding rule relies on "Weber's Law", which indicates that the change in a stimulus that will be just noticeable is a constant ratio of the original stimulus ${ }^{14}$, and may be expressed as:

$$
\frac{\Delta I}{I}=k
$$

where $I$ represents the initial intensity of a pixel, $\Delta I$ the change in the intensity of the pixel. $k$ signifies that the proportion on the left hand side remains constant despite the value of $I$ changing. In Eq. 8 and Eq. 9, $\gamma$ controls the trade-off between watermark visibility and watermark robustness within the image. The lower the value of $\gamma$ the less noticeable a watermark is within an image and therefore does not noticeably alter the perceived quality of the image ${ }^{15}$. For a more detailed description of the watermark embedding technique employed one may consult earlier work by the authors ${ }^{16-18}$.

\section{WATERMARK DETECTION TECHNIQUES}

A test statistic that is often employed in examining whether the image $I^{\prime}$ contains a watermark $w$ or not, is the correlation between the signal under investigation and the watermark:

$$
\rho=\frac{1}{3 N M} \sum_{i=1}^{N} \sum_{j=1}^{M} I^{\prime}(i, j) w(i, j)
$$

where $N$ is the image width, $M$ is the image height, $I^{\prime}$ is the possibly watermarked image and $w$ is the watermark sequence. This correlation value is compared to a suitably selected threshold $T$ and if the value of the correlation is greater than the threshold positive detection of the watermark is assumed. More detailed discussion on this type of watermark detection may be found in work by Barni et al. ${ }^{19}$ and Pitas et al. ${ }^{11}$ An example of a result from a correlation detection is shown in Fig. 6 and it can be seen that in the case of $a=3.96$ (the function seed to generate the watermark in this case) there is a peak in the correlation value which is above the value for the threshold and thus, one can say that the watermark is present in the image.

Statistical methods have also been used to determine if a watermark is embedded in a cover image or not. One such technique was presented by Nikolaidis and Pitas ${ }^{20}$. In this technique it is assumed that the watermark generated consists of an equal number of 0 and 1 pixels. The image can be divided into two subsets $A$ and $B$ :

$$
\begin{aligned}
& A=\left\{(n, m) \mid I_{n m}=1\right\} \\
& B=\left\{(n, m) \mid I_{n m}=0\right\}
\end{aligned}
$$

Once the generated watermark $w$ it is embedded into our original image $I$ using the additive technique, with an embedding factor of $\gamma$. The sample mean values of the pixels in the subsets $A$ and $B$ of the original image $I$ are calculated along with their standard deviation values $\left(s_{a}, s_{b}\right)$ :

$$
s_{a}^{2}=\frac{1}{(M \times N)-1} \sum_{n, m \in A}\left(x_{n m}-\bar{a}\right)^{2}
$$




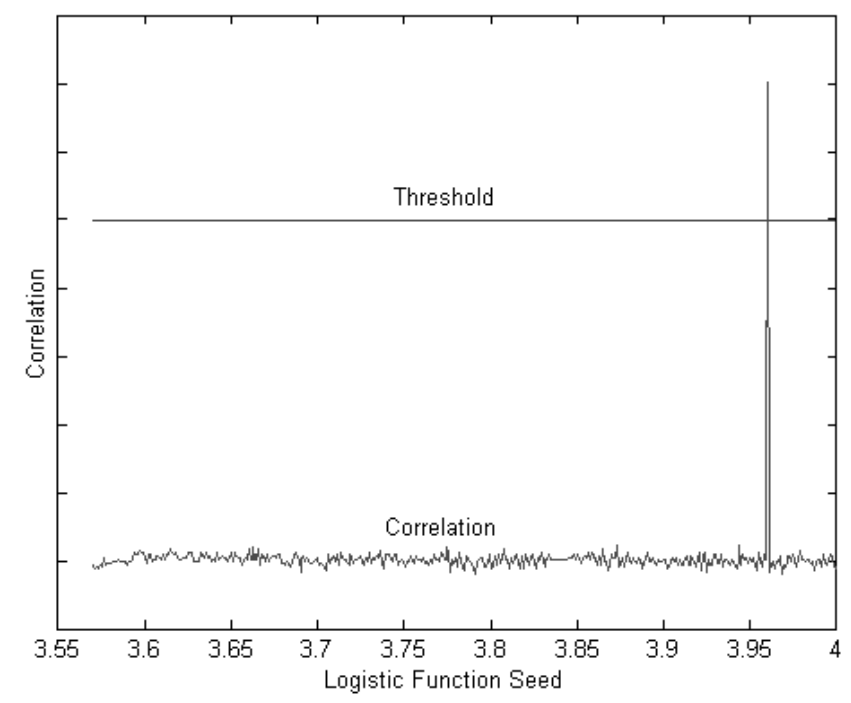

Figure 6. An example of a correlation detection result in the case where the embedded watermark was seeded with $a=3.96$.

$$
s_{b}^{2}=\frac{1}{(M \times N)-1} \sum_{n, m \epsilon B}\left(x_{n m}-\bar{b}\right)^{2}
$$

The sample mean value and the standard deviation of the pixels belonging to the subset $A$ of the possibly watermarked image $I^{\prime}$ are denoted by $\bar{c}$ and $s_{c}$ :

$$
\begin{gathered}
\bar{c}=\frac{1}{(M \times N)} \sum_{n, m \epsilon A} I_{n m} \\
s_{c}^{2}=\frac{1}{(M \times N)-1} \sum_{n, m \epsilon A}\left(I_{n m}-\bar{c}\right)^{2}
\end{gathered}
$$

Watermark detection is based on the examination of the difference $\bar{w}$ of the mean values $\bar{c}$ (pixels belonging to subset $A$ of the possibly watermarked image) and $\bar{b}$ :

$$
\bar{w}=\bar{c}-\bar{b}
$$

If the possibly watermarked image has been watermarked then $\bar{w}$ is close to the embedding factor, $\gamma$. However if the image is not watermarked or the image bears a watermark different to the one we are looking for, $\bar{w}$ is approximately zero.

Optical processing is faster than a similar digital technique due to the inherent parallelism of the system ${ }^{21}$ and this is the motivation for the use of an optical detection technique. The optical correlation technique used is based on the Matched Filtering Technique ${ }^{22,23}$ which has been shown to be an effective technique for watermark detection. For the optical detection system, the possibly watermarked image together with the watermark whose presence (or absence) in the image one wishes to determine are used as inputs to the detector. The watermark is used as the filter for the matched filter detector. The optical detection technique returns an output image which is the correlation between the possibly watermarked image and the watermark. An example of an output from the modelling of the optical detection technique is shown in Fig. 7. When the watermark being searched for is present in the possibly watermarked image there is a clear shape peak at the centre of the output, otherwise there is no peak at the centre. For more detail on the optical detection scheme employed one may consult earlier work by the authors ${ }^{16,17}$. 


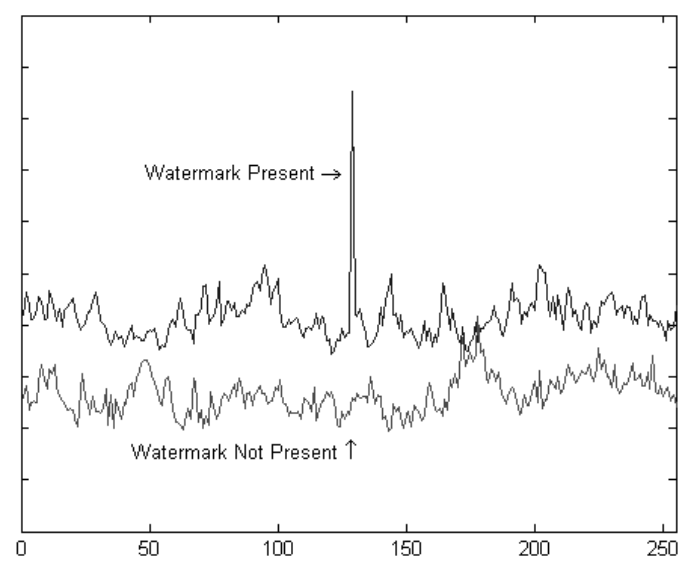

(b)

Figure 7. An example of an optical detection result in the case when there is a particular watermark present and also in the case where the watermark being searched for is not present in the image.

\section{LOWPASS AND HIGHPASS WATERMARKS}

By analysing the power spectrum one can determine whether a particular sequence is lowpass or highpass in characteristic. It was previously shown that for the logistic map, in general, as the seed increased more lowpass sequences are generated. This may be confirmed by comparison of the images generated using function seeds at the opposite ends of the chaotic regions, as shown in Fig. 8.
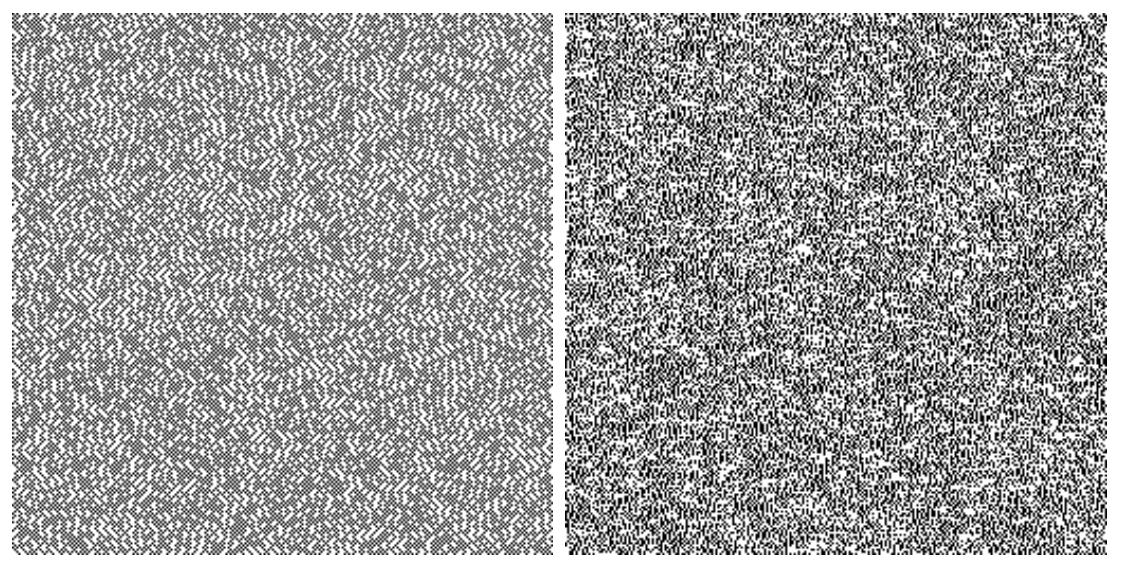

Figure 8. Watermark produced when the logistic function is seeded with (a) $a=3.6$ and (b) $a=3.9$.

In the case of $a=3.9$ (Fig. $8(\mathrm{a})$ ) there is much less density variation in watermark produced than in the case of the watermark generated when $a=3.6$ (Fig. 8(b)). This suggests that the watermark produced when $a=3.9$ is more lowpass than the watermark produced when $a=3.6$ and has shown to be the case ${ }^{17}$.

In the case of the Skew Tent Map (shown in Fig. 3) that as the value of the function seed increased more lowpass sequences are produced. For Bernoulli chaotic maps only lowpass sequences can be generated. The lowpass characteristics of these sequences decrease as the value of $n$ increases. This is unlike the chaotic sequences generated by both the logistic map and the Skew Tent Map as these mappings can produce both highpass and lowpass watermarks. The most lowpass sequence which may be generated with the Bernoulli Map occurs when $n=2$. 


\section{ATTACKS}

Images may be subject to two kinds of attacks, namely, intentional and unintentional attacks, such as Image Compression, Filtering, Noise Addition, Image Cropping, Multiple Marking, Removal Attempts and Desynchronization Attacks. In this paper we consider Noise Addition, JPEG Compression and Image Cropping, as these appear the ones most often discussed in the literature.

\subsection{Noise Addition}

Images processed by an optical system are often degraded by some random errors - this degradation is usually called noise and may occur during image capture, transmission or processing ${ }^{24}$. Noise may also be present as random background signals in transmission or communication signals. This "shot noise" is caused by the random fluctuations in the motion of charge carriers ${ }^{25}$, and may be modelled by a Poisson distribution. The Poisson distribution for mean $\lambda$ of a random variable $x$ is given by:

$$
p(x)=\frac{\lambda^{x} e^{-\lambda}}{x !}
$$

where $x$ is an integer and has the property that its variance $\sigma^{2}$ equals its mean $\lambda$. As Poisson noise is discrete for each pixel value the mean changes for each pixel and thus the variance of each pixel is different. We are interested in the performance of the optical detection scheme when a watermarked image is corrupted with noise, and have therefore, conducted experiments on watermarked images with various levels of noise intensity.

Watermarks generated using the Skew Map, Bernoulli Map and the Logistic Map were generated and subjected to varying levels of noise, and then tested for the presence of the watermark. The level of noise present in an image may be determined by calculating the Signal-to-Noise Ratio (SNR) value of the image. The SNR value is calculated using:

$$
S N R=20 \log _{10} \frac{\sum_{(n, m)} I(n, m)^{2}}{\sum_{(n, m)}\left(I(n, m)-I^{\prime}(n, m)\right)^{2}} \mathrm{~dB}
$$

where $I(n, m)$ is the original image and $I^{\prime}(n, m)$ is the noisy image. The lower the SNR value the more noise is present in an image. It was found that in the case of more lowpass watermarks for each map the more robust they were to the addition of noise. For example, in the case where the logistic map seeded with $a=3.9$ embedded watermarks were detected up to a value of $S N R=14.96 \mathrm{~dB}$. This compares well with the case where the generated watermark was created using the Skew Map with a function seed of 0.8. In this case correct correlation of the watermark occurred up to a $S N R=16.1 \mathrm{~dB}$. Fig. 9(a) shows an example of the cover image corrupted with noise, resulting in a SNR of $17.74 \mathrm{~dB}$.
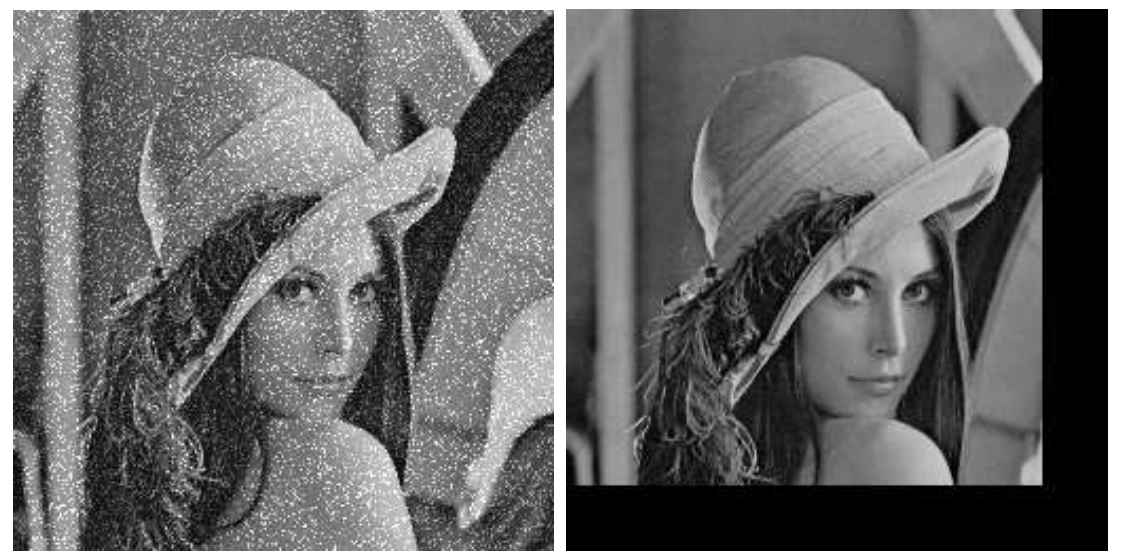

Figure 9. (a) Watermarked Cover Image (Fig. 1(a)) with a Signal-to-Noise level of $17.74 \mathrm{~dB}$ and (b) Cover Image cropped by $85 \%$ in both the horizontal and vertical directions. 


\subsection{JPEG Compression}

Watermarks generated using the Skew Map, Bernoulli Map and the Logistic Map were used to watermark the cover image shown in Fig. 1(a), and the watermarked image was subsequently subjected to JPEG compression. JPEG is a lossy compression technique designed to exploit known limitations of the Human Visual System (HVS), notably the fact that small changes in the image will go unnoticed when viewed by the human eye. It was observed that in general as the watermarks used had more lowpass characteristics increased robustness to JPEG Compression was observed. This is what is expected as lowpass watermarks have increased robustness with respect to image distortions that have lowpass characteristics (filtering, nonlinear filtering such as median filtering, lossy Compression etc. ${ }^{26}$. The results obtained in the case of the logistic function (e.g. when $a=3.9$ correct detection occurred up to a compression level of 18\%) were favourable in comparison to those for the Skew Map (e.g. when $\alpha=0.8$ correct detection occurred up to a compression level of $15 \%$ ) and Bernoulli Map(e.g. when $n=3$ correct detection occurred up to a compression level of $10 \%$ ).

\subsection{Image Cropping}

Image cropping refers to the process of removing a segment of the image. This attack is used to try to remove the segment of the image where the attacker may think the watermark is present. Watermarked images were subjected to image cropping of different degrees and then presented to the detector. An example of a cropped image (width $=85 \%$ of original width, height $=85 \%$ of original height) is shown in Fig. $9(\mathrm{~b})$.

It was found in the case of using the logistic equation for watermark generation that the results were favourable in comparison to the cases where the watermarks were generated using the Skew Map and the Bernoulli Map. For example, for all three mappings, correct detection occurred with a watermarked image which had been cropped by $85 \%$ of its width and $85 \%$ of its height. This is due to the fact that the watermark is embedded throughout the entire cover image and not just a select area of it.

\section{CONCLUSION}

Digital Watermarking is the process of embedding an identifying piece of data in a digital document. Chaotic functions have been suggested for the generation of watermarks and in this paper we review three such chaotic functions for their use in watermark generation. Skew Tent Maps and Bernoulli Maps are well defined maps and have previously been used for watermark generation. The logistic function is more ill-defined and we present an analysis of it and some important properties of it. These properties should be known to the person watermarking as they may produce non-chaotic periodic watermarks in the so-called "chaotic region". Various watermark detection techniques have been presented along with an optical detection technique. An image may be subjected to attacks (intentional or unintentional) and analysis on three of the most common were presented here. It was found that the logistic function performed equally as well, if not better, than the Skew tent and Bernoulli maps in the presence of these attacks. 


\section{REFERENCES}

1. I. J. Cox, J. Killian, T. Leighton, and T. Shamoon, "Secure spread spectrum watermarking for images, audio, and video," Proceedings of International Conference on Image Processing (ICIP'96) III, pp. 243-246, 1996.

2. A. Tefas, A. Nikolaidis, N. Nikolaidis, V. Solachidis, S. Tsekeridou, and I.Pitas, "Markov chaotic sequences for correlation based watermarking schemes," Proceedings of Chaos, Solitons and Fractals 17, pp. 567-573, 2003.

3. A. Nikolaidis and I. Pitas, "Comparison of different chaotic maps with application to image watermarking," Proceedings of IEEE International Symposium on Circuits and Systems, Geneva, pp. 509-512, 2002.

4. S. Tsekeridou, V.Solachidis, N.Nikolaidis, A.Nikolaidis, A. Tefas, and I.Pitas, "Bernoulli shift generated watermarks: Theoretic investigation," Proceedings of IEEE Int. Conf. on Acoustics, Speech and Signal Processing, pp. 1989-1992, 2001.

5. R. L. Devaney, A first course in Chaotic Dynamical Systems - Theory and Experiment, Perseus Books, Cambridge, Massachusetts, 1992.

6. M. Marek and I. Schreiber, Chaotic Behaviour of Deterministic Dissipative, Cambridge University Press, Cambridge, 1991.

7. K. Yang and M. Mills, "Fractal based image coding scheme using peano scan," Proceedings of ISCAS ' 88 1470, pp. 2301-2304, 1988.

8. R. Stevens, A. F. Lehar, and F. Preston, "Manipulation and presentation of multidimensional image data using the peano scan," Proceedings of IEEE Trans. Patterm Pattern Anal. Machine Intell, pp. 520-526, 1983.

9. A. Tefas, A. Nikolaidis, N. Nikolaidis, V. Solachidis, S. Tsekeridou, and I.Pitas, "Statistical analysis of markov chaotic sequences for watermarking applications," Proceedings of IEEE International Symposium on Circuits and Systems (ISCAS 2001), 2001.

10. A. Tefas, A. Nikolaidis, N. Nikolaidis, V. Solachidis, S. Tsekeridou, and I.Pitas, "Performance analysis of watermarking schemes based on skew tent chaotic sequences," NSIP'01.

11. S. Tsekeridou, V.Solachidis, N.Nikolaidis, A.Nikolaidis, A. Tefas, and I.Pitas, "Theoretic investigation of the use of watermark signals derived from bernoulli chaotic sequences," SCIA2001, 2001.

12. M. Schroeder, Fractals, Chaos, Power Laws - Minutes from an Infinite Paradise, W. H. Freeman, New York, 1991.

13. A. Lasota and M. C. Mackey, Chaos, Fractals and Noise - Stochastic Aspects of Dynmaics, Springer, London, 1994.

14. Encyclopaedia Brittanica Online, http://www.britannica.com/.

15. I. J. Cox, M. L. Miller, and J. A. Bloom, Digital Watermarking, Morgan Kaufmann, London, 2002.

16. A. Mooney and J. G. Keating, "Optical and digital technique for watermark detection," Proceedings of SPIE, Optical Information Systems 5202, pp. 97-105, 2003.

17. A. Mooney and J. G. Keating, "Noisy optical detection of chaos-based watermarks," Proceedings SPIE, Photonics North 5579, pp. 341-350, 2004.

18. A. Mooney and J. G. Keating, "The impact of the theoretical properties of the logistic function on the generation of optically detectable watermarks," Proceedings SPIE, Technology for Optical Countermeasures, Optics/Photonics in Defence and Security, London, pp. 120-129, 2004.

19. M.Barni and F. Bartolini, "Improved wavelet-based watermarking through pixel-wise masking," IEEE Trans. on Image Processing 10, pp. 783-791, 2001.

20. N. Nikolaidis and I. Pitas, "Robust image watermarking in the spatial domain," Signal Processing Elsevier 66, pp. 385-403, 1998.

21. J. Rosen, "Three-dimensional optical fourier transform and correlation," Optics Letters 22, pp. 964-966, 1997.

22. M. Shen, X. Zhang, L. Sun, P. J. Beadle, and F. H. Y. Chan, "A method for digital image watermarking using ica," 4th International Symposium on Independent Component Analysis and Blind Signal Separation , pp. 209-214, 2003. 
23. A. Sequeira and D. Kundur, "Communication and information theory in watermarking: A survey," Proceedings of Multimedia Systems and Applications IV, A. G. Tescher, B. Vasudev, and V. M. Bove, eds., Proc. SPIE 4518, pp. 216 - 227, 2001.

24. M. Sonka, V. Hlavac, and R. Boyle, Image Processing, Analysis, and Machine Vision-Second Edition, PWS Publishing, San Francisco, 1999.

25. F. R. Connor, Noise-Introductory Topics in Electronics and Telecommunication-Second Edition, Edward Arnold Publishing, London, 1982.

26. J. Fridrich, "Combining low-frequency and spread spectrum watermarking," Proc. SPIE Int. Symposium on Optical Science, Engineering and Instrumentation, pp. 2-12, 1998. 